\title{
ICSI with All Oocytes Recurrent Metaphase I Characterized by Absence Perivitelline Space
}

\author{
Yan Jiang*, Ge Song, Jingchuan Yuan, Xuhui Zhang \\ The Center for Reproductive Medicine and Infertility, Shijiazhuang Obstetrics and Gynecology Hospital Affiliated to Hebei \\ Medical University, Shijiazhuang, China \\ Email: ^13932117427@163.com
}

How to cite this paper: Jing, Y., Song, G., Yuan, J.C. and Zhang, X.H. (2021) ICSI with All Oocytes Recurrent Metaphase I Characterized by Absence Perivitelline Space. Open Journal of Obstetrics and Gynecology, 11, 1112-1116.

https://doi.org/10.4236/ojog.2021.119104

Received: July 28, 2021

Accepted: September 3, 2021

Published: September 6, 2021

Copyright $\odot 2021$ by author(s) and Scientific Research Publishing Inc. This work is licensed under the Creative Commons Attribution International License (CC BY 4.0).

http://creativecommons.org/licenses/by/4.0/

\section{(c) (i) Open Access}

\begin{abstract}
Previous cases reported recurrent all oocytes in repeated cycles were arrested at MI failed to mature after culture and ICSI resulted in total nonfertilization. Ionomycin induced first $\mathrm{PB}$ extrusion in patients with recurrent maturation-resistant MI oocytes. ICSI combined with assisted oocyte activation (AOA) on MII oocytes matured in vitro resulted in abnormal fertilization with no further embryo cleavage potential. The objective of this work was to research if AOA helpful to fertilization for recurrent maturation-resistant MI oocytes. In this case, patient both cycles retrievaled immature MI oocytes characterized by absence perivitelline space (APVS). In the second cycle, ionomycin was used for AOA after MI oocyte perfomed ICSI, no fertilization again.
\end{abstract}

\section{Keywords}

ICSI, Recurrent MI Oocyte Arrest, AOA, Ionomycin

\section{Introduction}

The oocytes classfied germinal vesicle (GV), metaphase I (MI) and metaphase II (MII) after controlled ovarian stimulation (COS) in in vitro fertilization (IVF). MI oocytes has capability to reach MII stage following in-vitro maturation (IVM) or fertilization, and blastocyst development performed with intracytoplasmic sperm injection (ICSI) immediately after denudation [1] [2] [3].

However, the cases previously reporting recurrent retrieval of all immature MI oocytes that were resistant to IVM [4] [5] [6] [7]. These MI arrest oocytes accompanied with immature fibrous appearance of the zona pellucida proceed further with ICSI resulted in total nonfertilization [5] [8]. Assisted oocyte activa- 
tion (AOA) has been shown to efficiently increase fertilization after ICSI in patients with low and total failed fertilization [9]. We present a case all oocytes at immature MI stage characterized by absence perivitelline space (APVS) with ICSI and AOA.

\section{Case Report}

A 28-year-old patient and her 30-year-old husband came to our clinic with a 3.5-year history of primary infertility. She had regular menstrual periods with a cycle length of 28 - 30 days, and was diagnosed to the left oviduct is obstructed, and the right side is blocked diagnostic by hysterosalpingography (HSG) in 2017 at a private hospital. A chromosomal analysis indicated a normal female chromosomal complement (46, XX). Her husband's semen parameters were in normal range. She underwent COS according to our routine IVF protocol. Antagonist protocol was started after doing baseline day 2 scan and hormonal profile. Day $2 \mathrm{FSH}$ was $5.74 \mathrm{mIU} / \mathrm{ml}$, LH was $3.16 \mathrm{mIU} / \mathrm{ml}$, estradiol was $44.75 \mathrm{pg} / \mathrm{ml}$. She was stimulated with uFSH 225 IU. Antagonist was started from day 7. She received 10 days of stimulation. Total gonadotrophin dose given was $2250 \mathrm{IU}$. Trigger was given on day 10 of stimulation with recombinant hCG, $0.25 \mathrm{mcg}$ subcutaneous. She had three follicles of $18 \mathrm{~mm}$, two follicles of $15-17 \mathrm{~mm}$ size on the day of trigger. Egg retrieval was performed after $36 \mathrm{~h}$ of trigger. A total of 5 oocytes were recovered by ultrasound guided transvaginal aspiration. They were cultured in the laboratory as per the standard protocol and short-time fertilizition. After cumulus removal, all the 5 oocytes were found to be arrested at MI stage of meiosis with APVS. Re-ICSI was done, absence of resistance to oolemma penetration during microinjection, and low ooplasm viscosity during aspiration. No fertilization and two degenerate were reported the next day.

The patient was taken for second cycle after 5-month interval. This cycles a long protocol of pituitary desensitization with triptorelin, in association with FSH and HMG. Dual trigger given with decapeptyl $0.2 \mathrm{mg}$ and hCG $0.2 \mathrm{mcg}$. On the day of trigger, she had three follicles of $18 \mathrm{~mm}$, five of $17 \mathrm{~mm}$ and six follicles of $16 \mathrm{~mm}$. Her E2 was $4616 \mathrm{pg} / \mathrm{ml}$, P $0.55 \mathrm{ng} / \mathrm{ml}$. OPU was planned after 36 $\mathrm{h}$ of trigger. This time 14 follicles were retrieved and all were in MI stage oocytes with NPVS again.

Because this patient recurrent all immature MI oocytes, and no fertilization in the first cycle. Approvoled by the couple, an hour after ICSI profomed, fourteen of these oocytes were exposed to $10 \mathrm{mM}$ ionomycin for $10 \mathrm{~min}$ for AOA. No fertilization again. The patient followed up in the work for two years, still not prengnency and consulted with donor oocytes.

\section{Discussion}

Approximately $17 \%$ - $20 \%$ of oocytes collected for IVF are meiotically immature, probably because of the stimulation of multiple follicles [5]. However, it is extremely rare for complete oocyte maturation failure to occur in IVF treatment 
[10]. Some cases reported all oocytes in repeated cycles were arrested at MI and failed to mature after culture. ICSI also resulted in total nonfertilization [4] [5]. At last, successfully conceived with donor oocytes [4].

Eichenlaub-Ritter U first reported the case of MI arrest in 1995 [11]. Bergere M reported MI arrest Patient with a family history with infertility [12]. The reason of MI arrest is unknown, underwent IVM and ICSI without further maturation or fertilization [13]. Abnormal cell-cycle control, Aberrant spindle structures with absence of microtubules dispersion of the female chromosomes or cytoskeletal function may be responsible for recurrent human MI oocyte arrest [5] [8]. Ultrastructural analysis demonstrated irregular chromatin condensation, disrupted spindle formation, and distorted and/or stunted microvilli protruding to the perivitelline space [10].

These MI maturation-resistant oocytes were observed zona pellucida (ZP) is immature, being narrow and fibrous, with a dense inner region [5]. Our research characterized with absence perivitelline space (APVS). Some study reported all oocytes with recurrent presenting narrow perivitelline space and heterogeneous zona pellucid (NPVS/HZP) and significant low oocyte maturity [14] [15]. Although lower MII oocytes rates, ICSI can fertilization and get pregnancy [14]. Absence of resistance to $\mathrm{ZP}$ and oolemma penetration, and low ooplasm viscosity during microinjection [15]. Because of NPVS/HZP, the polar body was usually difficult to differentiate, the MII oocytes rate may be not conclusive. How to identify the oocytes maturation stage without considering the polar body? What would happen if all of the oocytes without clear polar bodies were given ICSI [14]? We don't know if there were any relationship between these NPVS/HZP oocytes and our study MI arrest oocytes with APVS/IZP.

The MI oocytes from patients with a normal number of MII oocytes can fertilization and pregnancy proformed ICSI [16]. A higher proportion of MI oocytes progressed to MII after fertilization in mouse oocytes [17]. The MI oocyte can complete the extrusion of its PB after ICSI in human [16]. However, recurrent human MI oocyte arrest in owing to highly abnormal spindles, the chances of obtaining normal meiotic progression, or even normal fertilization and subsequent embryo development, are very low [8]. Lonophore induced first PB extrusion in MI oocytes from patients without maturation arrest but to a lower extent in maturation-resistant MI oocytes [8]. ICSI combined with AOA on MII oocytes matured in vitro or in vivo resulted in failed or abnormal fertilization with no further embryo cleavage potential [8]. We try to use of ICSI combined with $\mathrm{AOA}$ on $\mathrm{MI}$ oocytes in this study, resulted in unfertilization. Given the highly abnormal MI spindle formations and the lack of potential to overcome MI arrest using AOA by ionophore [8], more research is necessary to reveal the cause of these meiotic arrests.

Oocyte maturation failure: define the syndrome of oocyte maturation failure as a distinct oocyte disorder. At present, use of donor oocytes is the only option available for women with oocyte maturation failure if the defect is profound [7]. 


\section{Conflicts of Interest}

The authors declare no conflicts of interest regarding the publication of this paper.

\section{References}

[1] Strassburger, D., Goldstein, A., Friedler, S., Raziel, A., Kasterstein, E., Mashevich, M., et al. (2010) The Cytogenetic Constitution of Embryos Derived from Immature (Metaphase I) Oocytes Obtained after Ovarian Hyperstimulation. Fertility and Sterility, 94, 971-978. https://doi.org/10.1016/j.fertnstert.2009.04.035

[2] Li, M., Li, Y., Ma, S.Y., Feng, H.-L., Yang, H.-J., Wu, K.-L., et al. (2011) Evaluation of the Developmental Potential of Metaphase I Oocytes from Stimulated Intracytoplasmic Sperm Injection Cycles. Reproduction, Fertility and Development, 23, 433-437. https://doi.org/10.1071/RD10228

[3] Avci, B., Kasapoglu, I., Cakir, C., Ozbay, A., Ata, B. and Uncu, G. (2020) Fertilisation and Early Embryonic Development of Immature and Rescue in Vitro-Matured Sibling Oocytes. Human Fertility, 17, 1-10. https://doi.org/10.1080/14647273.2020.1714085

[4] Vandana, M. and Lakshmi, C. (2018) Unexpected Repeat Immature Oocyte Response after IVF Stimulation: A Case Report. Gynecological Endocrinology, 34, 100-102. https://doi.org/10.1080/09513590.2017.1369514

[5] Windt, M.L., Coetzee, K., Kruger, T.F., Marino, H., Kitshoff, M.S. and Sousa, M. (2001) Ultrastructural Evaluation of Recurrent and in-Vitro Maturation Resistant Metaphase I Arrested Oocytes: Case Report. Human Reproduction, 16, 2394-2398. https://doi.org/10.1093/humrep/16.11.2394

[6] Levran, D., Farhi, J., Nahum, H., Glezerman, M. and Weissman, A. (2002) Maturation Arrest of Human Oocytes as a Cause of Infertility: Case Report. Human Reproduction, 17, 1604-1609. https://doi.org/10.1093/humrep/17.6.1604

[7] Beall, S., Brenner, C. and Segars, J. (2010) Oocyte Maturation Failure: A Syndrome of Bad Eggs. Fertility and Sterility, 94, 2507-2513. https://doi.org/10.1016/j.fertnstert.2010.02.037

[8] Heindryckx, B., Lierman, S., Combelles, C.M., Cuvelier, C.A., Gerris, J., De Sutter, P. (2011) Aberrant spindle structures Responsible for Recurrent Human Metaphase I Oocyte Arrest with Attempts to Induce Meiosis Artificially. Human Reproduction, 26, 791-800. https://doi.org/10.1093/humrep/deq400

[9] Bonte, D., Ferrer-Buitrago, M., Dhaenens, L., Popovic, M., Thys, V., De Croo, I., et al. (2019) Assisted Oocyte Activation Significantly Increases Fertilization and Pregnancy Outcome in Patients with Low and Total Failed Fertilization after Intracytoplasmic Sperm Injection: A 17-Year Retrospective Study. Fertility and Sterility, 112, 266-274. https://doi.org/10.1016/j.fertnstert.2019.04.006

[10] Neal, M.S., Cowan, L., Louis, J.P., Hughes, E., King, W.A. and Basrur, P.K. (2002) Cytogenetic Evaluation of Human Oocytes That Failed to Complete Meiotic Maturation in Vitro. Fertility and Sterility, 77, 844-845.

https://doi.org/10.1016/S0015-0282(01)03257-5

[11] Eichenlaub-Ritter, U., Schmiady, H., Kentenich, H. and Soewarto, D. (1995) Fertilization and Early Embryology: Recurrent Failure in Polar Body Formation and Premature Chromosome Condensation in Oocytes from a Human Patient: Indicators of Asynchrony in Nuclear and Cytoplasmic Maturation. Human Reproduction, 10, 2343-2349. https://doi.org/10.1093/oxfordjournals.humrep.a136297 
[12] Bergere, M., Lombroso, R., Gombault, M., Wainer, R. and Selva, J. (2001) An Idiopathic Infertility with Oocytes Metaphase I Maturation Block: Case Report. $\mathrm{Hu}$ man Reproduction, 16, 2136-2138. https://doi.org/10.1093/humrep/16.10.2136

[13] Harrison, K.L., Sherrin, D.A. and Douglas Keeping, J. (2000) Repeated Oocyte Maturation Block. Journal of Assisted Reproduction and Genetics, 17, 231. https://doi.org/10.1023/A:1009401614028

[14] Li, M., Ma, S.Y., Yang, H.J., Wu, K.-L., Zhong, W.-X., Yu, G.-L., et al. (2014) Pregnancy with Oocytes Characterized by Narrow Perivitelline Space and Heterogeneous Zona Pellucida: Is Intracytoplasmic Sperm Injection Necessary? Journal of Assisted Reproduction and Genetics, 31, 285-294.

https://doi.org/10.1007/s10815-013-0169-9

[15] Sousa, M., Teixeira, D.S.J., Silva, J., Cunha, M., Viana, P., Oliveira, E., et al. (2015) Embryological, Clinical and Ultrastructural Study of Human Oocytes Presenting Indented Zona Pellucida. Zygote, 23, 145-157.

https://doi.org/10.1017/S0967199413000403

[16] Bilibio, J.P., Lorenzzoni, P.L., Meireles, A.J.C., Maciel, Y., Sales, P. and Costa do Nascimento, F. (2021) The Usefulness of Metaphase I Oocytes in Women Who Undergo Controlled Ovarian Hyperstimulation for Intracytoplasmic Sperm Injection. JBRA Assisted Reproduction, 25, 115-121.

https://doi.org/10.5935/1518-0557.20200062

[17] Yoon, J., Juhn, K.M., Yoon, S.H., Ko, Y. and Lim, J.-H. (2017) Effects of Sperm Insemination on the Final Meiotic Maturation of Mouse Oocytes Arrested at Metaphase I after in Vitro Maturation. Clinical and Experimental Reproductive Medicine, 44, 15-21. https://doi.org/10.5653/cerm.2017.44.1.15 